\title{
Calcific myonecrosis mimicking soft tissue sarcoma: A case report
}

\author{
YUICHIRO UKON $^{1,2}$, TAKAAKI TANAKA ${ }^{1,2}$, SHIGENORI NAGATA $^{3}$, HIROKI HAGIZAWA $^{2}$, YOSHINORI IMURA ${ }^{2}$, \\ HIRONARI TAMIYA ${ }^{2}$, KAZUYA OSHIMA ${ }^{2}$, NORIFUMI NAKA ${ }^{2}$, YASUAKI AOKI ${ }^{4}$ and SHIGEYUKI KURATSU ${ }^{1}$ \\ ${ }^{1}$ Department of Orthopaedic Surgery, Belland General Hospital, \\ Sakai, Osaka 599-8247; Departments of ${ }^{2}$ Orthopaedic Surgery and ${ }^{3}$ Pathology and Cytology, \\ Osaka International Cancer Institute, Chuo-ku, Osaka 541-8567; ${ }^{4}$ Department of Orthopaedic Surgery, \\ Japanese Red Cross Society Himeji Hospital, Himeji, Hyogo 670-8540, Japan
}

Received September 25, 2017; Accepted February 28, 2018

DOI: $10.3892 / \mathrm{ol} .2018 .8320$

\begin{abstract}
Calcific myonecrosis is a rare soft tissue condition. The first case was reported in 1960, however, the precise pathophysiology of calcific myonecrosis remains unclear. The disease was thought to arise from compartment syndrome within a confined space resulting in necrosis and fibrosis, subsequent repeated intralesional hemorrhage, mass enlargement and calcification. Several previous reports have described calcific myonecrosis, which include the formation of calcific myonecrosis after a prolonged period of post trauma. Notably, calcific myonecrosis has typically been described in the lower legs and characteristic imaging findings have been indicated. Furthermore, surgical intervention carries a high risk of complications. In the present case report 2 cases of calcific myonecrosis that occurred after a prolonged period of time following a traumatic event that impacted the lower leg were reported. CT images revealed disruption of calcified fascia and disease expansion into the outside of the fascia. Previous reports have implied that there is late focal enlargement of calcific myonecrosis following earlier enlargement, which may be caused by herniation through muscle fascia. However, no previous publications have focused on images for evidence of late local enlargement. To the best of our knowledge, this is the first report focusing on fascial herniation of calcific myonecrosis using images. Analysis of this feature using images may aid clinicians to differentiate calcific myonecrosis from malignancies.
\end{abstract}

\section{Introduction}

Calcific myonecrosis is a rare soft tissue condition that mainly occurs after a long period of time post trauma. It commonly

Correspondence to: Dr Takaaki Tanaka, Department of Orthopaedic Surgery, Osaka International Cancer Institute, 3-1-69 Otemae, Chuo-ku, Osaka 541-8567, Japan

E-mail: takaaki.tanaka.77@gmail.com

Key words: calcific myonecrosis, fascial calcification, fascial herniation occurs in the lower leg, most frequently in the anterior compartment, and is thought to be related to compartment syndrome. Malignancy is sometimes suspected by clinicians due to the large size and abnormal imaging features of calcific myonecrosis. Moreover, surgical incision of calcific myonecrosis carries a high risk of complications. As such, early diagnosis and appropriate management is important. Calcific myonecrosis is characterized by a slowly expanding soft tissue mass with necrotic tissue and calcification and was first reported in 1960 by Gallie and Thompson as a series of cases with past history of Volkmann's ischemic contracture (1). More than 60 cases of calcific myonecrosis have been reported to date, however, the precise pathophysiology of calcific myonecrosis remains unclear. The disease is thought to initially arise from compartment syndrome within a confined space resulting in necrosis and fibrosis based on histology (2), followed by repeated intralesional hemorrhage causing mass enlargement and calcification (3), then finally late focal enlargement of the mass, which is thought to be caused by herniation through the muscle fascia (4). However, there is no previous publication focusing on imaging of the enlargement process of calcific myonecrosis. Here, we report two cases with characteristic images that demonstrate fascial herniation, which may be involved in late focal rapid enlargement of calcific myonecrosis.

\section{Case report}

This study was approved by the ethics committee of our institutions. The patients provided informed consent regarding medical information and the publication of the present study.

Case 1. A 69-year-old woman with a history of left distal fibula fracture 46 years ago, which was treated with open reduction and internal fixation, noticed swelling of her lower limb. She had been asymptomatic and had not undergone any treatments for the past 20 years, but was referred to our outpatient clinic for investigation of a suspected malignant tumor detected on plain radiograph (Fig. 1A).

Physical examination showed a non-tender, elastic hard mass with normal temperature on palpation. There was restricted movement of the ankle joint (dorsiflexion $5^{\circ}$ plantarflexion $50^{\circ}$ ). Plain radiograph showed fusiform masses 
along the central to distal part of the lower leg (Fig. 1A). Computed tomography characterized the details of the fusiform masses, with calcified capsules broadly following the fascia. The contents of the fusiform masses comprised of homogeneous components and scattered calcification (Fig. 1B). The tibialis anterior (TA), extensor hallucis longus (EHL) and extensor digitorum longus (EDL) muscles were each replaced by a fusiform mass. The fusiform masses in TA and EHL penetrated the fascia between the muscles at the junction of the middle and distal thirds of the leg (Fig. 1B and C). An incisional biopsy was performed because there was a possibility of malignant tumor. The surface of the mass was covered in fascial tissue with thin calcification. The center of the mass was filled with a milky, low viscosity cloudy fluid containing yellow discoid solid material (Fig. 2A). Pathological examination showed amorphous deposition with calcification around the fascia (Fig. 2B). Two weeks after the biopsy, leg swelling had recurred, the surgical wound had not healed, and a sinus tract had formed. The patient continued to attend our outpatient clinic for 3 months to receive treatment for the wound.

Case 2. The patient is a 76-year-old man with a history of fractured right distal tibia and fibula from skiing 55 years ago. The fractures were treated with open reduction and internal fixation, but there was lower leg swelling after the injury that did not resolve for a long time postoperatively. Also, there was numbness in the distribution of the superficial and deep peroneal nerves, as well as weakness of right ankle dorsiflexion postoperatively.

After a period of 55 years without attending any medical institution, the man was referred to our outpatient clinic because of pain in the right anterior lower leg and growth of a mass lesion over 2 months.

Physical examination showed a non-tender, elastic-hard mass measuring $14 \times 17 \mathrm{~cm}$ in the anterolateral aspect of the right lower leg with normal temperature on palpation. Plain radiograph showed large fusiform masses with plaque like amorphous calcification (Fig. 3A). Computed tomography more clearly characterized the structure of the fusiform masses, demonstrating a central homogeneous component with scattered internal calcification covered by calcified capsules broadly following the fascia (Fig. 3B). The TA muscle was replaced by a fusiform mass, and the EHL and EDL muscles were also replaced by a single fusiform mass. The TA muscle capsule was disrupted by the fusiform mass in the junction of the middle and distal thirds of the leg with subcutaneous extension (Fig. 3C).

The patient was diagnosed with calcific myonecrosis, and opted for conservative therapy. However, the patient attended another institution because of increased fever and leg pain. The general practitioner diagnosed leg infection and performed incision and drainage. After the surgical procedure, swelling increased, calor and rubor appeared, and a draining sinus formed. The patient returned to our outpatient clinic due to worsening of these symptoms (Fig. 4A). A brown low viscosity exudate with light yellow discoid solid material was draining from the sinus (Fig. 4B). Pathological examination confirmed an inflammatory exudate with necrotic and focally calcified material (Fig. 4C). The exudate cultured positive for methicillin sensitive coagulase negative staphylococcus. Antibiotic treatment was required for 5 months and the patient also attended the outpatient clinic for wound care, however the wound had still not completely healed 1 year after incision.

\section{Discussion}

Calcific myonecrosis is a rare disease, which was first reported in two patients by Gallie and Thomson (1). More than 60 cases of calcific myonecrosis have been reported, however, the pathophysiological mechanism of these lesions remains incompletely understood. Evidence of muscle necrosis caused by compartment syndrome before calcific myonecrosis has been previously reported based on past history (1). Janzen et al proposed that decreased circulation caused by compartment syndrome within a confined space induces the initial muscle necrosis and fibrosis involved in calcific myonecrosis based on histology (2). O'Keefe et al postulated that repeated intralesional hemorrhage causes mass enlargement and calcification (3). Several features of calcific myonecrosis have also been previously reported. First, calcific myonecrosis occurs a long time after the initial typically traumatic event, which commonly involves fracture and acute compartment syndrome. The average time period between the initial event and presentation to hospital with calcific myonecrosis across 50 cases is 37.2 years, with a range of 6-59 years. The average age of presentation is 61.0 years, with a range of $37-87$ years (2-26). Second, calcific myonecrosis often occurs in the lower leg (92\%, 54 of 59 cases) (2-9,11-30). Regarding the lower legs, fusiform masses most frequently occupy the anterior compartment $(85 \%, 41$ of 48 legs), followed by the lateral compartment (38\%, 18 of 48 legs) and the deep posterior compartment (27\%, 13 of 48 legs), which is consistent with the compartments where chronic compartment syndrome commonly occurs (2-9,11-19,21-25,28-31). In these compartments, pressure promptly elevates and local blood flow tends to be chronically impeded, which are favorable conditions for disease enlargement. Third, calcific myonecrosis has a characteristic appearance on radiograph, demonstrating fusiform masses with plaque like amorphous calcification. The calcifications are usually linear and sheet-like along muscles and compartments. Smooth bony erosions are sometimes seen, and are better characterized on CT $(4-7,9,14-16,18,19,22,25,30)$. The fusiform masses are covered by calcific capsules, with a center filled by a homogeneous component and small scattered calcifications. The masses replace muscle, sometimes entire compartments. Fourth, surgical incision of calcific myonecrosis often causes complications such as sinus tract formation and infection, leading O'Dwyer et al to propose that calcific myonecrosis should be considered as a 'don't touch lesion' (4). However, infection and sinus tract formation from skin puncture caused by trauma may have already occurred by the time of presentation. $(5,7,11,21,23,26)$. It is important to inform the patient of these risks when considering conservative treatment. There are several reports of excisional biopsy and complete resection with skin flap reconstruction for calcific myonecrosis, however there was a high rate of complications such as sinus tract formation (35\%, 11 of 31 cases) and infection (26\%, 8 of 31 cases) $(2,3,5-7,11,12,15-18,20-23,26,27)$. Our 2 cases also had these four features, similar previous reports. There was a long period between the initial trauma event and presentation with calcific myonecrosis in both our cases (46 and 55 years). 

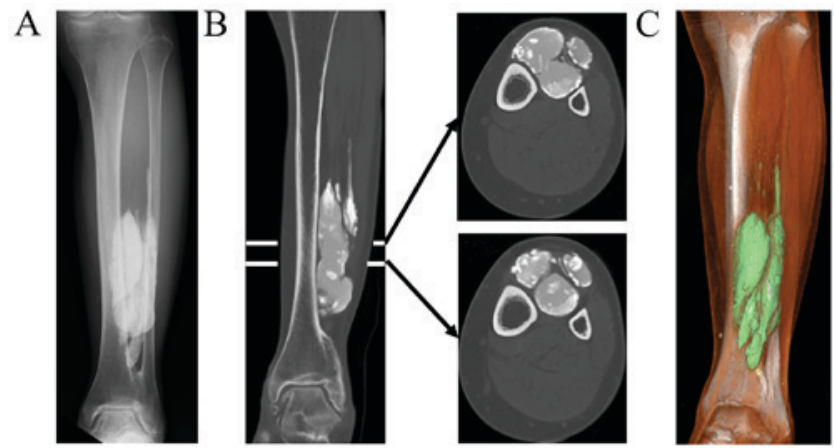

Figure 1. Case 1 images. (A) Anteroposterior radiograph of the left leg showing fusiform masses in the anterior compartment. Computed tomography showing fusiform masses. The left image is a coronal view and (B) right images are axial views cut in the horizontal lines of the left image. Reconstruction of computed tomography showing (C) bone and disease and muscles in a $3 \mathrm{D}$ image.

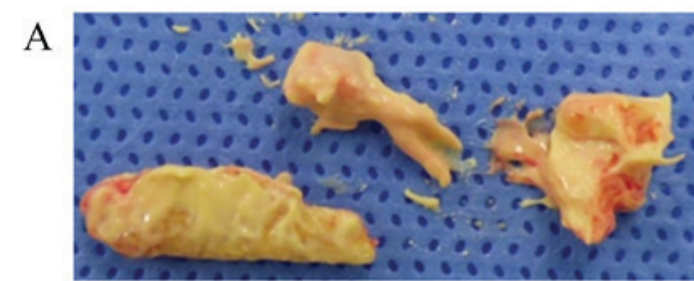

B

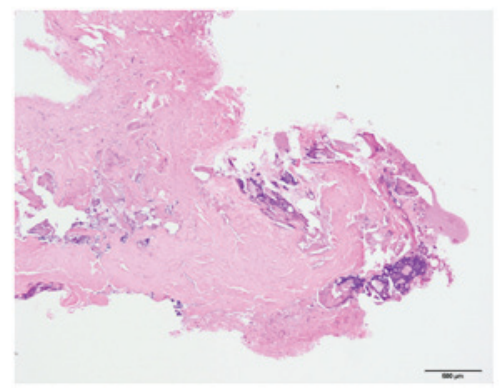

Figure 2. Incisional biopsy of Case 1. (A) Yellow discoid solid material inside the lesion. (B) Pathological examination showing amorphous deposition with calcification around the fascia; low-power view (magnification, $x 4$ ).
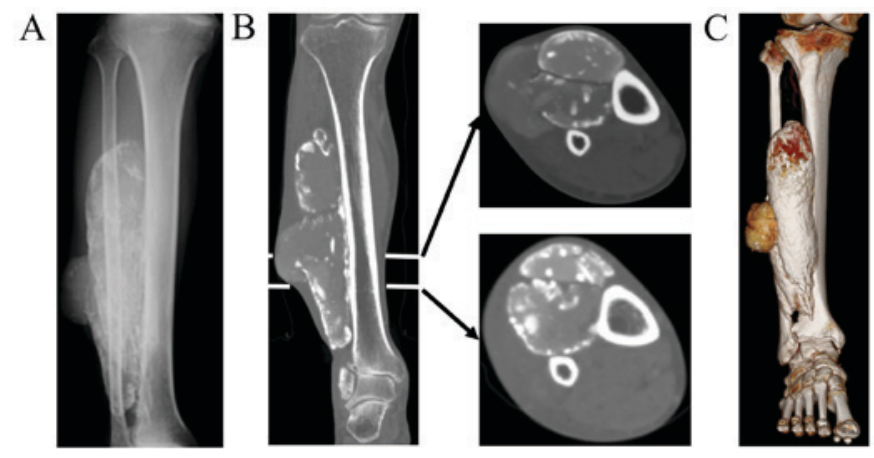

Figure 3. Case 2 images. (A) Anteroposterior radiograph of the left leg showing fusiform masses in the anterior compartment. Computed tomography showing fusiform masses. The left image is a coronal view and (B) right images are axial views cut in the horizontal line of the left image. (C) Reconstruction of computed tomography showing bone and disease in a 3D image.

Both occurred in the lower leg anterior compartment, and in both cases disease replaced the TA, EHL and EDL muscles. There were severe complications in both cases, with delayed

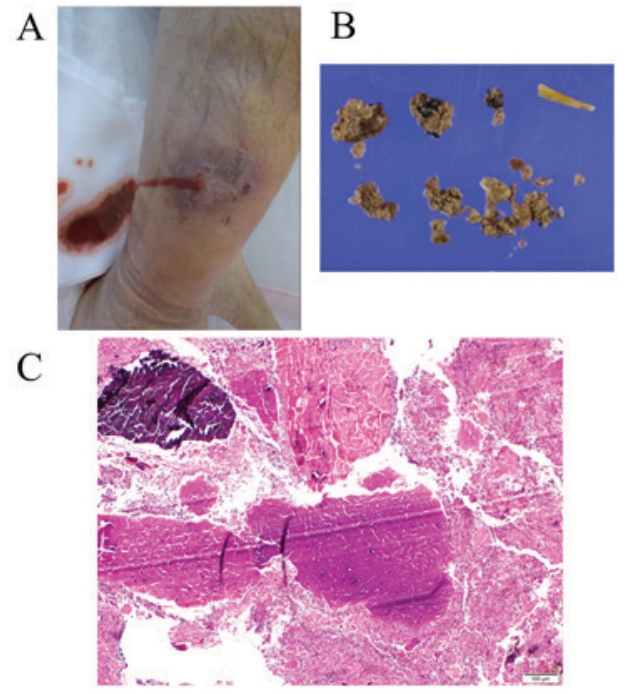

Figure 4. A draining sinus and material from the sinus in case 2. (A) Picture of the right distal leg with swelling and rubor. (B) Light yellow discoid solid material draining from the sinus. (C) Pathological examination showing inflammatory exudate with necrotic and focally calcified substance; high-power view (magnification, x40).
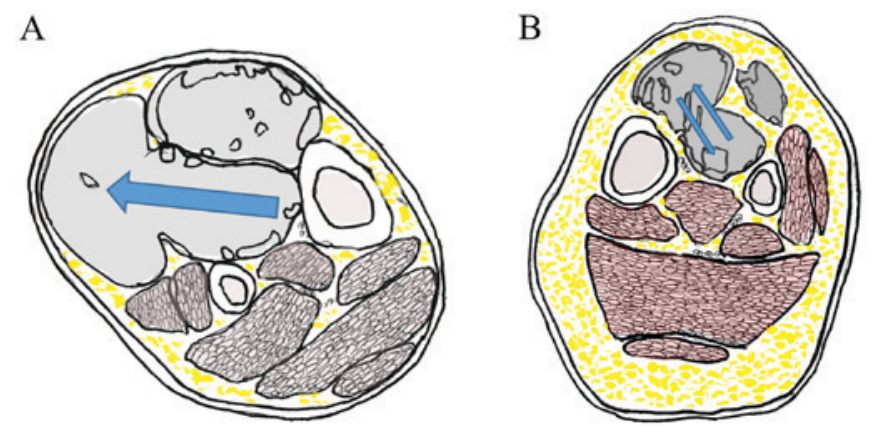

Figure 5. Diagrammatic illustration of disease expansion. (A) Case 1 and (B) Case 2 .

wound healing after excisional biopsy in Case 1, and infection and delayed wound healing after incision in Case 2. Further, our two cases exhibited a new feature of calcific myonecrosis. O'Dwyer et al proposed that late focal enlargement occurs after early enlargement occurs in calcific myonecrosis. They suspected that this might be caused by herniation through muscle fascia (4), however, there is no report with evidence specifically for fascial herniation. Rupture of the calcified fascia and disease expansion into other layers was observed on CT images in Case 2 (Fig. 5). This suggests that late rapid enlargement occurred when the patient noticed growth of the mass lesion 2 months before the referral. Calcified fascia defect was also observed on CT images in Case 1, which may be related to the late enlargement process. The ruptured calcified lesions in both cases were situated at the junction of the middle and distal thirds of the leg, consistent with the location where fascial hernias commonly occur in chronic compartment syndrome, thought to be sites of mechanical weakness (31). We presume that disease replacing muscle along the fascia occurs and subsequently disease replacing compartments occurs as a result of repeated fascial herniation, eventually forming huge masses replacing entire compartments of the lower leg, such as 
in previous reports $(22,24)$. This new feature on images may help clinicians to differentiate this disease from malignant tumor. The differential diagnosis of this rare condition with soft tissue calcification includes malignant tumor such as synovial sarcoma, epithelioid sarcoma, parosteal osteosarcoma, or periosteal sarcoma. These conditions sometimes appear similar to calcific myonecrosis on images because they can be within muscle, however, if there is a calcified fascial herniation this may favour the diagnosis of calcific myonecrosis rather than malignancies.

Our study has several limitations. One limitation is the images, which were obtained only after the patient was referred. Fascial herniation was demonstrated on these images, however it is unclear when and how the herniation occurred. The relationship between fascial herniation and late focal enlargement is also uncertain. A longer follow-up period is needed to observe the process of enlargement. Another limitation is that there are very few previous reported cases of calcific myonecrosis, such that significant and consistent imaging features are not yet evident. Past reports considered that the time period between the initial event and presentation to hospital with calcific myonecrosis may affect occurrence. On the other hand, there is the possibility that ageing itself after the initial event is related to occurrence. Statistically, there is also a correlation between the time period to presentation and age [correlation coefficient $=0.668, \mathrm{P}<0.05$ (Spearman's rank correlation coefficient)]. It is difficult to accurately determine if age itself plays a role in occurrence of calcific myonecrosis. Prospective research is needed to elucidate the underlying mechanism.

In conclusion, our two cases demonstrate a new feature of calcific myonecrosis on images. Rapid focal enlargement of calcific myonecrosis may occur, and is potentially caused by herniation through the muscle fascia. This new feature on images may help clinicians to differentiate this disease from malignancies.

\section{Acknowledgements}

The authors would like to thank Dr Matthew Lukies from the Department of Radiology,Alfred Health (Melbourne,Australia) for the English proofreading of this manuscript.

\section{Funding}

No funding was received.

\section{Availability of data and materials}

The data generated during the current study are not publicly available due to the privacy of the patients but are available from the corresponding author on reasonable request.

\section{Author's contributions}

YU collected the patient data and was a major contributor in writing the manuscript. TT organized the study and assisted in writing the manuscript. SN performed the histological examination of calcific myonecrosis specimens. YU, TT, YA and SK treated the two patients presented in this manuscript. YA and SK also contributed to data collection, interpretation, discussion and critical review of the manuscript. All other authors (HH, YI, HT, KO and NN) contributed to data collection, interpretation, discussion and critical review of the manuscript. All authors read and approved the final manuscript.

\section{Ethics approval and consent to participate}

This study was approved by the ethics committee of our institutions.

\section{Consent for publication}

The patients provided informed consent regarding medical information and the publication of the present study.

\section{Competing interests}

The authors declare that they have no competing interests.

\section{References}

1. Gallie WE and Thomson S: Volkmann's ischaemic contracture: Two case reports with identical late sequelae. Can J Surg 3: 164-166, 1960.

2. Janzen DL, Connell DG and Vaisler BJ: Calcific myonecrosis of the calf manifesting as an enlarging soft-tissue mass: Imaging features. AJR Am J Roentgenol 160: 1072-1074, 1993.

3. O'Keefe RJ, O'Connell JX, Temple HT, Scully SP, Kattapuram SV, Springfield DS, Rosenberg AE and Mankin HJ: Calcific myonecrosis. A late sequela to compartment syndrome of the leg. Clin Orthop Relat Res 318: 205-213, 1995.

4. O'Dwyer HM, Al-Nakshabandi NA, Al-Muzahmi K, Ryan A, O'Connell JX and Munk PL: Calcific myonecrosis: Keys to recognition and management. AJR Am J Roentgenol 187: W67-W76, 2006.

5. Yuenyongviwat V, Laohawiriyakamol T, Suwanno P, Kanjanapradit $\mathrm{K}$ and Tanutit P: Calcific myonecrosis following snake bite: A case report and review of the literature. J Med Case Rep 8: 193, 2014.

6. Sreenivas T, Nandish Kumar KC, Menon J and Nataraj AR: Calcific myonecrosis of the leg treated by debridement and limited access dressing. Int J Low Extrem Wounds 12: 44-49, 2013.

7. Portabella F, Nárvaez JA, Llatjos R, Cabo J, Maireles M, Serrano C, Pedrero S, Romero E, Pablos O and Saborido A: Calcific myonecrosis of the leg. Rev Esp Cir Ortop Traumatol 56: 46-50, 2012 (In Spanish).

8. Jalil R, Roach J, Smith A and Mukundan C: Calcific myonecrosis: A case report and review of the literature. BMJ Case Rep 2012: pii: bcr2012007186, 2012.

9. De Carvalho BR: Calcific myonecrosis: A two-patient case series. Jpn J Radiol 30: 517-521, 2012.

10. Rynders SD, Boachie-Adjei YD, Gaskin CM and Chhabra AB: Calcific myonecrosis of the upper extremity: Case report. J Hand Surg Am 37: 130-133, 2012.

11. Papanikolaou A, Chini M, Pavlakis D, Lioni A, Lazanas M and Maris J: Calcific myonecrosis of the leg: Report of three patients presenting with infection. Surg Infect (Larchmt) 12: 247-250, 2011.

12. Chun YS and Shim HS: Calcific myonecrosis of the antetibial area. Clin Orthop Surg 2: 191-194, 2010.

13. Freire $M$ and Simonelli PS: Your diagnosis? Calcific myonecrosis. Orthopedics 33: 461-521, 2010.

14. Peeters J, Vanhoenacker FM, Camerlinck M and Parizel PM: Calcific myonecrosis. JBR-BTR 93: 111, 2010.

15. Papanna MC, Monga P and Wilkes RA: Post-traumatic calcific myonecrosis of flexor hallucis longus. A case report and literature review. Acta Orthop Belg 76: 137-141, 2010. 
16. Okada A, Hatori M, Hosaka M, Watanuki M and Itoi E: Calcific myonecrosis and the role of imaging in the diagnosis: A case report. Ups J Med Sci 114: 178-183, 2009.

17. Muramatsu K, Ihara K, Seki T, Imagama $\mathrm{T}$ and Taguchi $\mathrm{T}$ : Calcific myonecrosis of the lower leg: Diagnosis and options of treatment. Arch Orthop Trauma Surg 129: 935-939, 2009.

18. Constantine S, Brennan C and Sebben R: Calcific myonecrosis: Case report and radiopathological correlation. Australas Radiol 51: B77-B81, 2007.

19. Dhillon M, Davies AM, Benham J, Evans N, Mangham DC and Grimer RJ: Calcific myonecrosis: A report of ten new cases with an emphasis on MR imaging. Eur Radiol 14: 1974-1979, 2004.

20. Larson RC, Sierra RJ, Sundaram M, Inwards C and Scully SP: Calcific myonecrosis: A unique presentation in the upper extremity. Skeletal Radiol 33: 306-309, 2004.

21. Holobinko JN, Damron TA, Scerpella PR and Hojnowski L: Calcific myonecrosis: Keys to early recognition. Skeletal Radiol 32: 35-40, 2003.

22. Wang JW and Chen WJ: Calcific myonecrosis of the leg: A case report and review of the literature. Clin Orthop Relat Res 389: 185-190, 2001.

23. Jassal DS, Low M, Ross LL, Zeismann M and Embil JM: Calcific myonecrosis: Case report and review. Ann Plast Surg 46: 174-177, 2001.

24. Zohman GL, Pierce J, Chapman MW, Greenspan A and Gandour-Edwards R: Calcific myonecrosis mimicking an invasive soft-tissue neoplasm. A case report and review of the literature. J Bone Joint Surg Am 80: 1193-1197, 1998.

25. Ryu KN, Bae DK, Park YK and Lee JH: Calcific tenosynovitis associated with calcific myonecrosis of the leg: Imaging features. Skeletal Radiol 25: 273-275, 1996
26. Snyder BJ, Oliva A and Buncke HJ: Calcific myonecrosis following compartment syndrome: Report of two cases, review of the literature, and recommendations for treatment. J Trauma 39: 792-795, 1995.

27. Nagamoto H, Hosaka M, Watanuki M, Shiota Y, Hatori M, Watanabe M, Hitachi S and Itoi E: Calcific myonecrosis arising in the bilateral deltoid muscles: A case report. J Orthop Sci 22: 790-794, 2017.

28. Karkhanis S, Botchu R, James S and Evans N: Bilateral calcific myonecrosis associated with epilepsy. Clin Radiol 68: e349-e352, 2013.

29. Finlay K, Friedman L and Ainsworth K: Calcific myonecrosis and tenosynovitis: Sonographic findings with correlative imaging. J Clin Ultrasound 35: 48-51, 2007.

30. Batz R, Sofka CM, Adler RS, Mintz DN and Dicarlo E: Dermatomyositis and calcific myonecrosis in the leg: Ultrasound as an aid in management. Skeletal Radiol 35: 113-116, 2006.

31. Fraipont MJ and Adamson GJ: Chronic exertional compartment syndrome. J Am Acad Orthop Surg 11: 268-276, 2003.

This work is licensed under a Creative Commons Attribution 4.0 International (CC BY 4.0) License. 